\title{
EducActiveCore: Computational Model to Educational Personalization Based on Multiagent and Context-Aware Computing
}

\author{
Fernão Santos \\ Mackenzie Presbyterian University \\ São Paulo, SP, Brazil \\ Pollyana Notargiacomo \\ Mackenzie Presbyterian University \\ São Paulo, SP, Brazil
}

\begin{abstract}
With the growth of online courses and, usage of mobile access allowing students execute educational activities in multiple locales, with variety of data and media content, new perspectives of educational support using different computing models can be observed. Some of most recent evolved computing models stand out in areas like Social Networks Analysis, Artificial Intelligence, Mobile Computing and Context-Aware Computing. Understanding the combination of these computing areas as complementary researches, this work investigates the applications of these computing technics to modeling an intelligent computational engine with educational personalization purposes. In this resume of a research in progress, a reduced implementation prepared as proof of concept simulating aspects of the target model, operates as centralized adaptive engine. The implemented engine, applied Artificial Neural Networks on classification tasks and routing recommendation. A group of 27 students participated in an experiment interacting with the adaptive engine using a mobile application provided. The mobile application allowed tracking of interface during usage flow by students, and provided to students the adaptive engine recommendation results. Around $59 \%$ of students confirmed the recommendation effectiveness of adaptive engine. In this experiment, at the end of each participation, students sent feedbacks about application features. The current results indicate the viability of computational model related to automation of classification tasks to environment identification and activity routing recommendation. In brief, the initial experiment presented encouraging results, indicating that the continuity of research could result in a useful tool to online educational platforms.
\end{abstract}

Keywords: Artificial Neural Networks, Artificial Intelligence, ContextAware Computing, Multiagent Systems. 


\section{INTRODUCTION}

This research investigates the elements of an artificial intelligent model to support the personalization of educational process. Currently, with growing mobile computing adoption, students involved in educational process can perform activities interacting with a variety of application platforms, devices and physical environments in different locales. Considering the growth of online curses, this scenario creates an opportunity and a requirement to increase the availability of educational resources in different locations and appropriate time. To realize it in a proper manner, educational environments and resources should be mapped and analyzed to correctly identify and qualify its relevance to students within learning process. An automated and adaptive computational model can be applied to perform this process.

The basic behavior expected to this model should vary according to specific student's needs and experience on each environment accessed and depends on student's profile interacting with the model. This intelligent computational model comprehends collect and process information from various student's perspectives, including data of urban mobility, social networks, educational activities, environments frequented and educational resources.

In a concrete example, if a student performs educational tasks interacting with social networks on a bookstore and change location to a library or university, this change of location can reflect on type of media provided as content in social network accessed. Beyond the adaptability of content provided, the model manages useful information regarding availability of physical resources on a given environment, like 3D printers and other educational equipment under reservation or limited usage polices in locations intended to be visited by students. In current technological scenario, the most common computing interaction to activate and coordinate similar behavior could be through mobile and wearable devices connected on wireless networks and in mostly cases activating different applications. Considering the possibilities of variety models of applications in student's interaction, the unconnected flow of information and unmanaged process of activation between these applications, can distract the student from specific and relevant educational content.

The computational model proposed in this research covers an alternative approach to support reduction of student time loss on content mapping and collection. Another aspect of proposed model is automated planning of educational tasks and its optimized route. The optimized route allows anticipate technical setup tasks with environments while students moves from a locale to another.

This article introduces the basic concepts of this work in progress, the research proposing an adaptive computational model structured to support student's interactions with context-aware environments. Considering the research comprehensiveness, this paper highlights the scope of optimized routing and the process of context-aware interaction. Results of first experiments involving this scope are presented. This article summarizes the others complementary concepts 
covered by research.

The content of this paper is organized in the following structure: An introduction to Context-Aware Computing is presented on Section II. Multiagent Systems and Context-Aware Computing are introduced in a correlated domain at Section III. A macro view of proposed EducActiveCore platform and the its principal decomposition and objectives is presented in Section IV. Initial conclusions and next phases are introduced on Section V.

\section{CONTEXT-AWARE COMPUTING: DEFINITION, MODELS AND APPLICATIONS}

\section{A. Definition and Computing Models}

Context-Aware computing models can be described essentially by computational environments that can adapt itself and current context based on user presence and interactions (Henricksen, et al., 2006), without explicit user supervision or intervention to this adaptive process (Baldauf, et al., 2007). There is no a final definition and rigid boundary to specify what compose a contextaware environment. Authors refer to key characteristics most appropriate to their research to define resources and services of a context. Some common descriptions of these resources are presented as information describing and characterizing a person or object in interaction with computer application (Abowd, et al., 1999). Beyond the informational aspects, physical elements and temporality of available resources (Bellavista, et al., 2012) went published as features that are central to define a context.

Previous researches (Schilit, et al., 1994) resumed the context computing as a result on combination of three information domains regarding on where computational resources are, what kind of resources is provided and, who is using it. Modeling these characteristics in attributes, import and exchange it in form of relevant information to identify a context is a key process to support the context computing adaptive process (Makris, et al., 2013). The changing process of a context started by sensors, network events, mobile device location detection or the combination of all these examples, provides several new different set of attributes to describe the context and its related resources.

\section{B. Context-Aware Learning Application}

To start the base reference of this research, works on context-aware computing applications within educational and learning domains, helps to define the objective and scope of this research central model. Investigations and development on context-aware applications to support learning process can be found on domains like language learning (Ogata, et al., 2004), using environment recognition to apply a compatible vocabulary according to user's location, supported by mobile devices and highlighting the importance of mobile devices to interact with context and evolving the application to detect the collaborative situational needs (Ingersoll, et al., 1989). Another example based on context-aware computing implementing an instructional application (Cheverst, et al., 2000) is built to identify tourist locations, relevant content and help tourist 
guides to take decisions considering the best matches for the group of visitors. Considerations of mobile device relevance within these tourist guide assistant and how embedding the solution on mobile devices to turn invisible and accessible to users was introduced. On collaborative learning, previous research investigates learning environment built on a peer-to-peer architecture (Yang, S. J., 2006) and demonstrate the applicability of a common protocol (using metadata structure) to determine context resources identification, information extraction and interoperability. The structure of meta-data, proposed in form of context-aware learning environment ontology, is processed with an adaptive model to evolve the ontology with aim to expand the interoperability.

\section{Multi-Agent Systems AND CONTEXT-AwARe COMPUTING}

Commonly, Context-Aware Computing considers the self-managed configuration. Previous investigations with Agent systems applied to ContextAware Computing, covered e-commerce business domains applying intelligent negotiation through case base reasoning and context history analysis (Kwon, et al., 2004). Related to user context personalization, frameworks based on Internet webservices, Internet address identification and segmentation proposes frameworks to act on behalf of users on pre-selection and definition of context content and services before turn it available to users (Kwon, et al., 2005). References regarding technical aspects to support multiconnected environments using agent-based model (Soldatos, et al., 2007) helps to establish this work base. Related researches covering the orchestration needs performed by multiple agents to process individual tasks adapting it behavior according to a collective result (Aouatef, et al., 2014) indicates an applicability of the concepts together. Previous research presented a specific approach proposing adaptive content to Digital TV environment applying the multi-agent model on personalization of educational process (Santos, F. R. D., 2010). Diverse domains of applicability with multi-agent and context-aware computing establish support to this current research and, mainly, those that directs the investigation to educational models.

\section{EDUCACTIVECORE: AdAPTIVE SUPPORT TO STUDENT'S INTERACTIVITY WITH CONTEXT-AWARE COMPUTING}

Research Proposal and Objectives

The main objective of this research is propose an intelligent computational model to provide a multimodal tool to support student's interactivity with context-aware environments during educational process. The multimodal concept considered in this research is regarding to student's and educator's perspectives attended by this model, named as EducActiveCore.

Observed from the perspective of a tool to education domain experts, this model has the objective to be an additional tool to acquire information about data related to learning tasks elaborated by other educators, content recommended to students through automated process and expert's designation. As complementary objective, is to help educators to identify how this content and the mode of its access impacts and influence the result of learning process 
performed by students. Based on shared data, collected and processed by this model, educators can select elements from shared set of tasks and student's profiles to reuse or adjust these elements to compose a new task. This new task composition must be perceived by adaptive model through processing (applying machine learning process) and, as result of this processing, the model can suggest to educator a new alternative on task composition.

From the centric perspective on students, adaptive support involving learning process emerges with the combination of context-aware computing and artificial Intelligent Agents acting on learn profiling and personalization of student's interactions with tasks execution. Observed within this objective, the model performs a similar usefulness of an analytics system specific to process educational data. This approach delimits the boundaries of main behavior proposed: intelligent recommendation, being active and adaptive on student's perspective. Active aspects foreseen to this model performs identification on what learning tasks students are involved, searching and classifying relevant content and resources to establish and, finally, identify the appropriate approach to provide it to students. Complementary active aspect is context-aware interaction. Beyond the actual digital consuming profile of students with multiconnected and decentralized (Rosaci, D., \& Sarné, G. M., 2010) computer interaction behavior, moving its attention from screens like Smart TVs, using console games, tablets, smartphones and mobile devices, all those devices interconnected and providing convergent media content, the wearable devices and context-aware computing are evolving rapidly.

Adaptive aspect in this model processing, applied in the combination of all these personal computing technologies makes sense to facilitate the access to educational resources in right place, time and format aiming fine profile personalization. It can turn available a new perspective to understand the student's learning behavior and allow educators acquire new point of view on how student's location moving pattern, device changing and content selection reflects on learning results. Observing this student's behavior as an object containing the perspective of student's navigation flow, reinforces the need of fine personalization and characterization like previous researches covered (Silva, L., et al., 2006).

Connected applications have been developed under convergence perspective of content and user interface familiarity. Usability and computer human interaction aspects are constantly reviewed considering multi screen access (Viel, C. C., et al., 2013), normally covering mobile screens (smartphones and tablets), desktop and large screens (Smart TVs and digital displays) and, recently, the wareable devices (smartwhatch and connected glasses). Concerning the learning application aspects, this variety should be observed in its usefulness and effectiveness.

Related to this problem, one approach proposed to support this research, is to perform the observation on how student's behavior transitioning through different environments, using devices to access educational content and, moving 
from several distinct connected environments, influences on performance results. The focus on this point considers the environments context-aware supported. One of the main reasons is the ability to provide connection services to learners and, hence, interconnection capabilities allowing exchange information with this proposed platform.

\section{Model Definition and Description}

The central point of this research, apply different and complementary computational models, since Genetic Algorithms to Artificial Intelligent Agents. Thin boundaries and interoperability needs of the macro components idealized, leads this research to propose a modular organization (Figure 1), initially structured by responsibility domains.

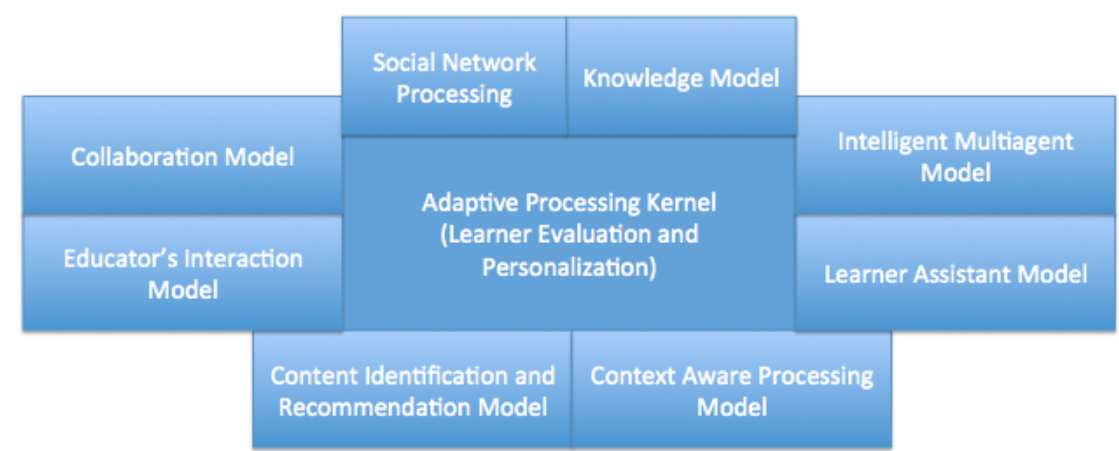

Figure 1. Overview of domain composition.

Figure 1 contains an organization of main structural components foreseen. The short summary of each component and its objective in the research is described.

\section{Social Network Processing}

Social Network platforms are key facilitators to stimulate learners sharing (Chow, W. S., \& Chan, L. S., 2008) knowledge and interaction within groups. Aggregated to this view, emerges multiplicity of mobile educational applications (Ally, M. (Ed.)., 2009) socially connected. The Social Network Processing is initially proposed as an engine to act as abstraction hub, aggregating the diversity of attributes regarding student's collaboration preferences. Different from the other components of EducActiveCore platform, that are threated as models, which can be subdivided in several engines or complementary models, this engine does not predicts evolve by itself. It necessary must attend the selected social networks to establish interoperability.

\section{Collaboration Model}

Collaboration Model is defined in the scope of this research as an engine to manage and promote the process of sharing attributes and metadata of educational tasks. Being a premise for this research conception, the shareable characteristic of a given collection of attributes and metadata related to task composition and performance results, are assembled with a set of additional attributes indicating the level of readiness to reuse. The importance of this 
engine as complement on Educator's Interaction Model processing is to perform cyclic analysis on tasks, identifying which group of tasks reach the level of completeness to compose the result on a query for similarity. With this additional processing centered in collaboration model, complex filtering algorithms involving this operation can be modified and evolve the engine independently from Educator's Interaction Model.

\section{E. Educator's Interaction Model}

Educator's Interaction Model manages amount and variability of data collection generated by observation, interactivity and tracking of student's activity. It provides to educator an analytic view, allowing use the result to tracking in fine granularity level the flow performed by learners as input to create new activities based on parameters generated from previous tasks. It enables the computational model to be used as a task templates repository to educators, allowing starting a new task with reusing the existents through querying or dynamically recommended. The applicability of Kernel processing is considered as support on task creation or task template extraction, to help educators reach the right match for defined purposes.

\section{F. Content Identification and Recommendation Model}

As intrinsic part of adaptability and personalization process performed in conjunction with Kernel and Multiagent Model, is content identification, filtering and recommendation as main result from these combined process. Considering the delimited objectives and the modularity proposed to this research, the model responsible for recommendation can act as complimentary on overall processing. The interaction between Recommender Model and Kernel, occurs under a qualitative evaluation of information recommended to learner. This qualitative evaluation is based on a fit indicator of information relevance to learner considering a specific domain. An example of evaluation with fit indicator within a specific domain, can be described by a recommendation with a set of information mapped in an educational task witch student's performance threshold is considered satisfactory. If student's performance threshold is not reached and identified as unsatisfactory, the fit indicator is applied to content indicating a lower priority on reuse for similar educational tasks.

\section{G. Learner Assistant Model}

The main objective of Learner Assistant Model is turn available to students an interactive tool to receive notifications, improve the dynamic of communication with other users and adjust personal profile information and preferences of platform usage. The processing from Kernel and Agent model are presented to learners as a result from combination of Kernel process synchronized with local Agent and resulting in a new state for local Agent and an updated state for notifications, messages and an eventual adjustment of interface that represents Learner Assistant model. 


\section{H. Knowledge Model}

Knowledge Model isolates adaptive kernel's algorithms from operations to mining data and the data persistence manipulation process, grouping all related operations into this module. The concepts to persist, search and retrieve the correct data used in each component in proposed model makes sense to be reunited in unique model to abstract the complexity from diverse resources used by the intelligent modules provided and, consequently, its generated data.

\section{Adaptive Processing Kernel}

This is the main model proposed on this research, containing the collection of algorithms acting as unique artificial intelligent engine. The aim of this kernel is identify the proper combination of student's behavior with computing interaction and, execute the orchestration of the complementary models to increase student's performance results on educational tasks.

Each complementary model proposed to interoperate with the Kernel, can decrease the algorithm implementation needs on Kernel and allows specific points of structure evaluation, adjust and replacement according to research's evolving and enhancement process.

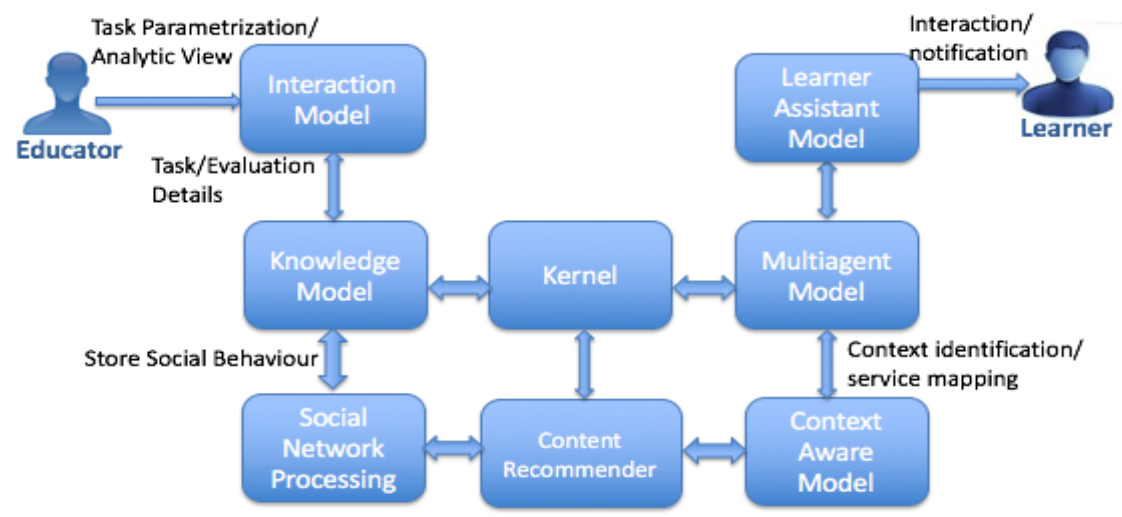

Figure 2. Processing coordinated by Kernel.

Figure 2 contains an illustration exemplifying the flow of macro interoperability foreseen between components. In this simple draft, without any precision and details of all operations expected, the arrows indicate which modules communicates directly with each other. Observing the illustration, the arrows indicates interaction flows between Kernel and Multiagent Model, and between Multiagent Model and Learner Assistant Model. This example indicates that there is no direct interaction between Learner Assistant Model to Kernel without Multiagent Model intermediation. Decouple central Kernel algorithms from operations of complementary components (case of storing and retrieving data which is an operation of Knowledge Model), contributes to rapidly reorganize the Kernel algorithms, data flow and evolve it independently of changes on support modules due to replacement and adjust. This strategy keeps the scope of Kernel in its boundary that is adaptive and predictive support on learning process. 
Processing the prediction of best match with diverse student's specific requirements and interaction elements, technological environment, context aspects and the frequent changes involved in this process, needs continuous parameterization and adjustment. With computing models requiring constantly human intervention or supervision on data and parameter input, this adjustment process could be inefficient. Starting from initial large amount of attributes and related values and, determining the coefficients to direct the processing to appropriate results through interactive process are inherent characteristics of computing applying machine learning models.

The set of algorithms in this Kernel, is organized to perform precisely mining and tracking on data collected from the complementary models. Knowledge Model provides the mining and tracking process, allowing the deep navigation in data collected, on proper format and structure depending on how the Kernel algorithms performs the query. This set of algorithms is divided into two groups of distinct operation; Students Evaluation and Students Personalization.

\section{1) Students Evaluation}

Within Student's Evaluation operation, the algorithms collect student's data results related to educational tasks and determine their acceptance according to specific parameters. These parameters, initially established on Educator's Interaction Model, evolve by combination of different computational technics, highlighting the application of methods adapted from Genetic Algorithms (Mitchell, M., 1998). Considering application of crossover operation from genetic algorithms, a sub selection of attributes from a parameter, combined with a second sub selection of attributes from another parameter, compose the new resultant parameter. An exhaustive analysis of each attribute contained on a given parameter to identify throughout comparison steps what could be the best for this case, possibly decreases the performance and effectiveness of algorithm. An internal organization for parameters and its classification according processing objectives, permits an identification of attribute influence due its labelled mark and position inside parameter.

The parameters, are composed by complex attributes collection (for contextservice representation example). This similar internal organization of parameters is applied to other parameter classes (student's profile, path followed to execute the defined educational task etc.) considering its specific characteristics and application. To determine the next enhanced parameters in relation to those covered on previous iteration which global result was indicated as not compatible with the criteria of acceptance, subsets of attributes from previous parameters (especially those indicated as locally positive to process) are selected and combined on a new parameter.

In this scenario, indicate the attribute's local performance implies to apply a fitness index to each attribute evaluated (locally and globally) and store this data to increase the historical knowledge base for intelligent mining and extraction.

Following the example of context-service representation, from student's 
perspective, the usefulness result of this process is provide a new logical and optimized suggestion on how take advantage of the resources provided by current context identified or even the location mapped as next destination. Identifying what is the next location and, hence, context-service available as other related personal preference anticipation depends on complementary processing performed by group of personalization algorithms.

\section{2) Students Personalization}

The group of algorithms responsible for Student's Personalization performs its operation in alternate mode: In Self-operation mode and, in Cooperation mode. Self-operation mode occurs evaluating and monitoring student's interaction behavior on context environments, social networks, content recommendation and collaboration model, forming a Frame of Student's Profile.

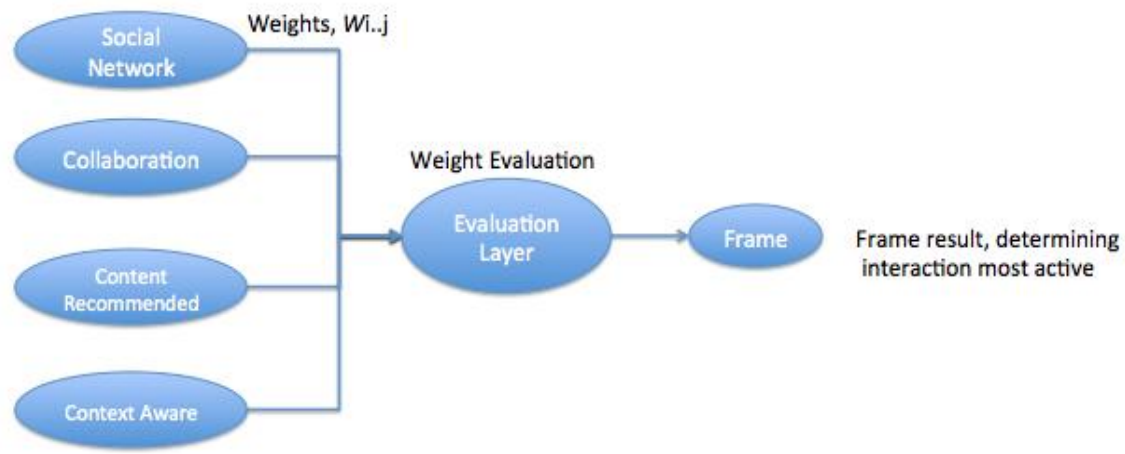

Figure 3. Process of Frame definition.

Figure 3 illustrates the macro process of Frame definition, considering the different models foreseen. Attributes of interactions being normalized to weights and submitted to an evaluation process can indicate which model is predominant at the instant of Frame composition. In cooperation mode, in response of Student's Evaluation process, this mode performs a cycle correlating student's task performance with the frame obtained. This scenario is expected mainly on educational task with incremental evaluation.

Tracking the Frame, allows the evaluation process to identify its recurrence and variability, hence, the influence on student's task performance. If evaluation process detects a Frame recurrence, and correlates it to a task results with lower performance, this Frame (attributes and predominant configuration) will be marked with an indicator of performance ranking (lower in this case) and postponed on the future suggestion on similar cases. The algorithms used to determine the Frame, are based on Artificial Neural Network models, specifically a backpropagation model (Basheer, I. A., \& Hajmeer, M., 2000). A crude analogy between artificial neural network models and the cooperation mode, permits associate the messages received from student's evaluation process with neurotransmitter to activate a new iteration to update Frame identification process. 
Is not scope of this research apply integrally and rigorously genetic algorithms models and operations used as reference (Mitchell, M., 1998) and all artificial neural networks technics (Basheer, I. A., \& Hajmeer, M., 2000). The machine learning models theory and the algorithms elaborated in this research to compose kernel's evolving process, must attend implementation according to expected operation on research.

\section{J. Intelligent Multiagent Model}

To support students in interaction process, a Multiagent Model is proposed to act as local personalized assistant. For the purpose of this research, as a solution using context aware computing, becomes useful local processing with the set of records with last actions executed and, with minimal data used to compute the current action according to student's perspective. The concept of local processing in this scenario, refers to Multiagent operation independent of interconnection with Kernel. This Multiagent Model is foreseen to frequently communicate online with Kernel but emerges as necessary due to common issues related to complementary resources like data repository based on cloud platforms and its availability (Alshwaier, A., et al., 2012) or while a network connection is not available for example.

The aim of applying Intelligent Multiagent Model in this research is to simplify the different local computation models. From student's utility view, the Agent Model can be available as embedded application on personal connected devices searching for available connections and service discovering on a context-aware environment according to student's profile and needs.

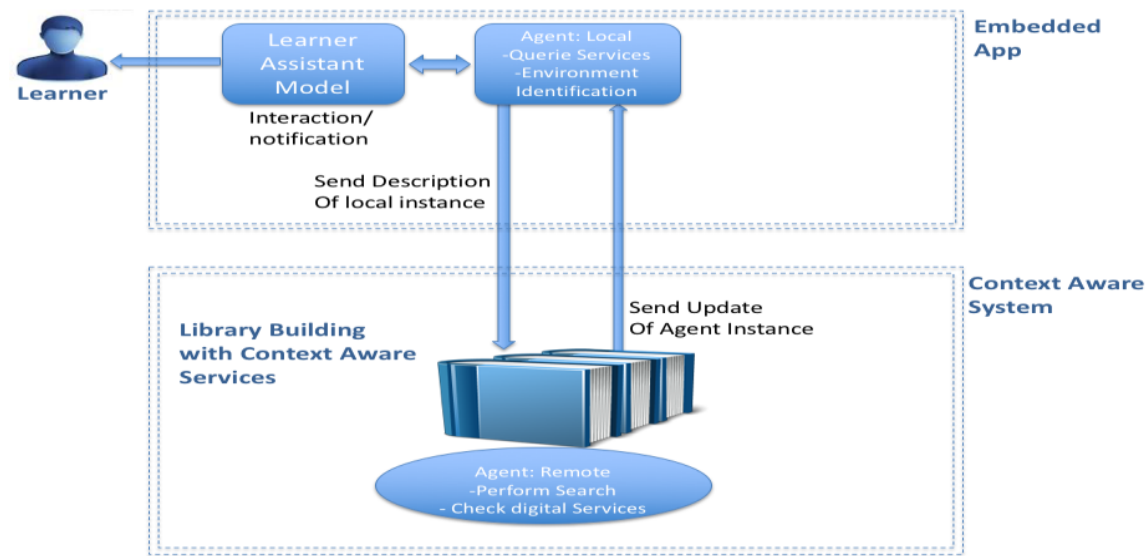

Figure 4. Multiagent system as protocol interaction

The Figure 4, illustrates the macro view of interaction with a local instance on embedded application and its remote replica. The same Agent representation can be transported to an identified context-aware environment (in this case, acting as a protocol) and perform different computation using local resources from environment instead of consuming local processing from student's device. This strategy reduces the problems related to device's energy efficiency and other questions that could affect student's experience with embedded application containing Agent Model. 
Considering this approach to use the student's local Agent as a protocol to communicate with context-aware environments, allows device's embedded application to send data related to student's profile and educational tasks where this learner is involved, previous activities performed and its results, next activities and all other necessary data used by local Agent to execute its processing

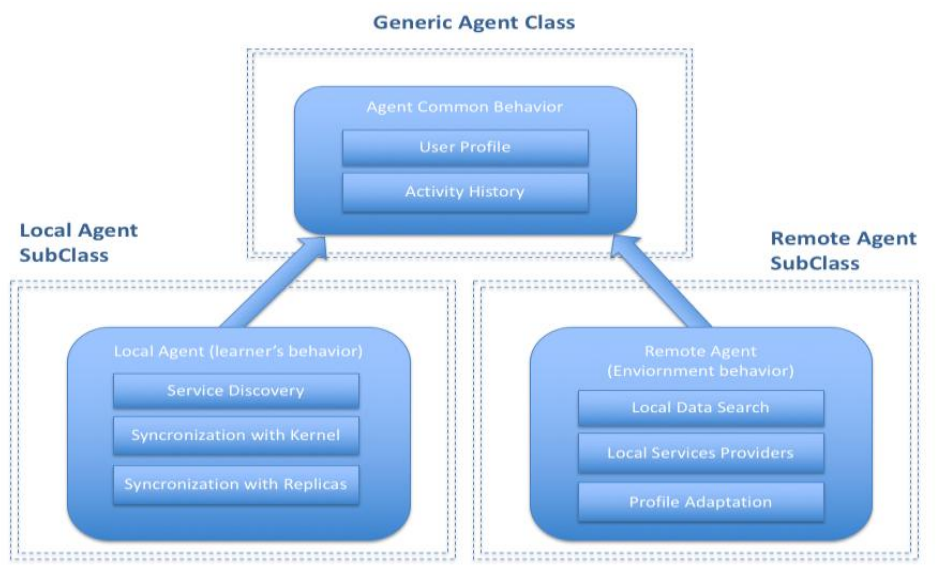

Figure 5. Agent Model specialization

An example of differences and specialization levels of each Agent model is illustrated on Figure 5. This set of data, representing the student's local Agent, creates another instance of Agent on context-aware environment. This new instance, is described in this research as remote Agent, due its additional behavior to process according to environment model using initial state received as input parameters. Specialization applied to each Agent model attends the variability aspects of context-aware environments where some attributes and behavior related to student's profile is shared to perform remote Agent tasks and, considering environment characteristics, specific behavior and attributes are inherent of processing. Tasks performed by remote Agent on context-aware environment, return to student's device as a result to update and merge behavior with device's agent instance and reflect its results on Kernel synchronization and Learner Assistant Model.

\section{K. Context Aware Model - Service Discovery and Resource Identification}

Considering the objectives of this research, the basic concept of context aware model must contain resources provided by a combination of physical location identification, descriptors and its digital services, observing the student's profile and needs. The model composition of services and resources are intrinsically characteristic to environment accessed by learner. A university laboratory can provide a 3D printer. A bookstore allows access on its book catalog. A commercial center indicates the store with specific scholar supplies. These distinct examples of environment differ on purpose and may differ on its characterization but they should present similarity with other environments under same objective classes. 
Organizing these different group of environments classes in a common vision, consolidates the need of an interpretation where a service discovery and resource identification process must abstract the variability of each class implementation details from student's local Agent. Conceptually observing the local Agent behavior on Service Discovery and Resource Identification interaction, some common elements from context-aware platforms definition (Zimmermann, A., et al., 2007) emerges as necessary to insure the abstraction level and reduced complexity model applied to local Agent.

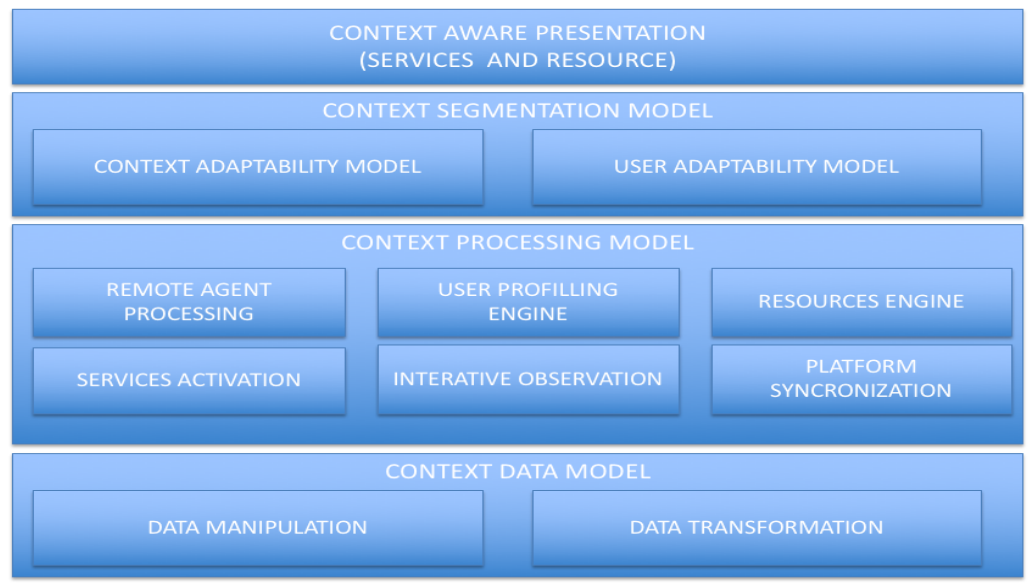

Figure 6. Context Aware Platform organization

The Figure 6 illustrates this research vision with a macro organization of components proposed to establish a context-aware computing environment. The upper layer Context Aware Presentation, communicate with local Agent to present the service discovery and resource identification. The service discovery, provides the catalog of available services in environment. Operations operation of resource identification, permits the compatibility verification with a local Agent's action intent related to a determined service previously discovered. Segmentation Model layer contains the management of user adaptability that control the level of interaction with learner. The context adaptability uses this level of interaction as parameter to calibration and setup with learner without interaction history.

In an overall and integrated example, if a local Agent queries and discovers an available 3D print service, resources engine verifies if the necessary drivers and supplies are compatible to attend the print request for student's project. If an incompatibility between service and requisition is detected, the resources engine could be update the available drivers to attend the request, if applicable. Eventually, a request for supplies reposition can be started if it turn insufficient. In case of impossibility with supplies reposition or driver's updates, the context segmentation configures the unavailability of this service specifically to user or group of users, and reflects it on presentation model to avoid new requests to same service. 


\section{PRELIMINARY EXPERIMENTS AND RESULTS}

Introducing the problem and scope of initial experiment

The algorithms regarding Multiagent and Kernel computational models proposed in this research, interacting with context-aware environment, are focus of initial feasibility study, and composes the first part of experiment. Due to expected variability of locales in interaction with students, becomes necessary identify and classify these locales not only based on their descriptors or identifiers provided. Given this scenario, an automated process to classify and recommend the environments mapped by Kernel, where students can access and perform educational activities, is an interesting point of analysis. A group of algorithms was elaborated to compose this second part of initial experiment.

\section{Structure of Experiment}

Considering the broad effort to implement all necessary algorithms and infrastructure foreseen in this research, a reduced set of functionalities and computational resources was combined to simulate a fraction of overall model described previously. At current stage of this research in progress, the adaptive computational model proposed has a minimum flow of its logic implemented as an experimental platform to proof of concept. This flow consists in data processing to simulate classification tasks related to student's educational activities and interactions with context-aware environments: Classification of Environment and Routing Recommendation to student's activities. Routing Recommendation includes the implementation of mobile application to simulate the basic Multiagent operation.

The research will analyze different intelligent computing architectures. In this experiment based on classification task, was applied Artificial Neural Networks. Classification process involves assigning objects into determined groups or classes based on a number of observed attributes related to those objects. The objective of experiment as proof of concept is not exhaustively compare the different architectures of neural networks and its variants (Samarasinghe, S. 2016). A neural network with Multi Layer perceptron (MLP) architecture was selected to experiment. This type of network is widely used for pattern classification, recognition, prediction, approximation and problems which are not linearly separable (Maren, A. J., et al., 2014).

The structure of this experiment consists in a group of algorithms implemented to establish Kernel classification tasks using MLP Neural Networks. Distinct implementations of MLPs was used on this classification tasks. The implementation of MLPs was used to classify locales, and a set of complementary specific algorithms was implemented to execute routing recommendation and, to simulate interactivity with context-aware environments. A group of volunteer's (university students) from distance education regular courses were invited to test the platform in parallel with their real use situations. The tracking of mobile application interface used by student's during platform tests was collected. The macro description of main process implemented during experiment are described as follows: 
Kernel Classification Task: Determining the class of Locale

The following main steps of MLP neural network implementation and execution was executed:
A. Obtain/prepare dataset with attributes and records of experiment and test
B. Normalize dataset
C. Select training set to classification tasks
D. Implement neural network to classification
E. Train the networks
F. Test the networks

The primary classification task, will indicate the class of context aware locale in student's current interaction where educational activities and their related contents are accessed and performed. In this initial experiment, the classes of environments went distinguished in two basic classes: Class of Educational Environment (EE) and Class of General Use (GU). The EE class refers to locale with specific educational and research purposes, including schools, libraries, universities research centers. The GU class, groups other locales with commercial or public services not specific to educational objectives.

Obtain and Prepare Data Set

An educational attributes collection used as reference was obtained in http://equipment.data.ac.uk/. This data collection, forms a database aggregating equipment information across a number of UK universities and contains over 24.000 records and contains 27 attributes. The original set of attributes in this dataset after pre-processed and filtered to this experiment purposes, was reduced to following 8 attributes:
1. Type
2. Name
3. Description
4. Related Facility
5. ID
6. Technique
7. Location
8. Contact Name

To elaborate a quality list of real attributes regarding General Use locales that are compatible and complimentary to attributes previously mapped, the list of attributes was based on https://www.wbdg.org/space-types/joint-use-retail. This reference contains structured descriptors to build common spaces and its related standards of architectures and services. These attributes used in the classification process are:
9. Entry Display
10. Cashier Counter
11. Display
12. Customer Service 
13. Representatives

14. Manager Office

15. Staff Workstations

16. Lavatory

17. Store Room

18. Housekeeping

Considering a data repository that conforms with this list of attributes, the sample records of this experiment was obtained from https://coworkingbrasil.org. It is a platform that aggregates information about Brazilian cowering spaces from diverse finalities and characteristics, including commercial and educational spaces. Performing queries in this platform for diverse available places, resulted in approximately 720 records to generate a initial dataset according to attributes defined. An attribute indicating this previously know class (EE class or GU class) of records was added indicating its category: 19. Environment.

\section{Normalizing Data Set}

Attributes of dataset went normalized in range from 0 to 1 depending on it completeness and relevance in educational purposes. Considering the attributes of dataset in priority order, attributes from 1 to 8 has more relevance to determine if locale belongs to EE class. For example, the attribute ID on this dataset, contains the URL address of real photo related to equipment/resource indicated on record. Since this attribute contains verifiable data of resource, it receives the value 1 in normalization process. If data in attribute points to an incorrect image or non URL (absence of $h t t p$ prefix or '.' indicating a domain, for example) a lower value than 1 was applied, and if no data available, 0 was used. With dataset prepared with know sources of records, two output attributes added at end of dataset indicates the class. This is useful to prepare training data and validate the network after training process.

\section{Creating Training Set}

In this coworking dataset, there is 350 records stablished as a main training set to this experiment, distributed in 200 records of GU class and 150 records with educational class (including scholar public spaces).

\begin{tabular}{|l|c|c|}
\hline \multicolumn{3}{|c|}{ Training Data distribution } \\
\hline \multicolumn{1}{|c|}{ CLASS } & Total Records & $\%$ Training set \\
\hline EE & 150 & $43 \%$ \\
\hline GU & 200 & $57 \%$ \\
\hline
\end{tabular}

Table 1. Class distribution

Table 1 contains the records distribution of main training dataset. Additionally, a set of 30 records (15 of each class) was selected to form a Test Set. A collection of records to validate the MLP neural network after training. 


\section{Implementing Neural Network}

To first classification task, a MLP neural network was implemented to attend attributes mapped as neurons inputs.

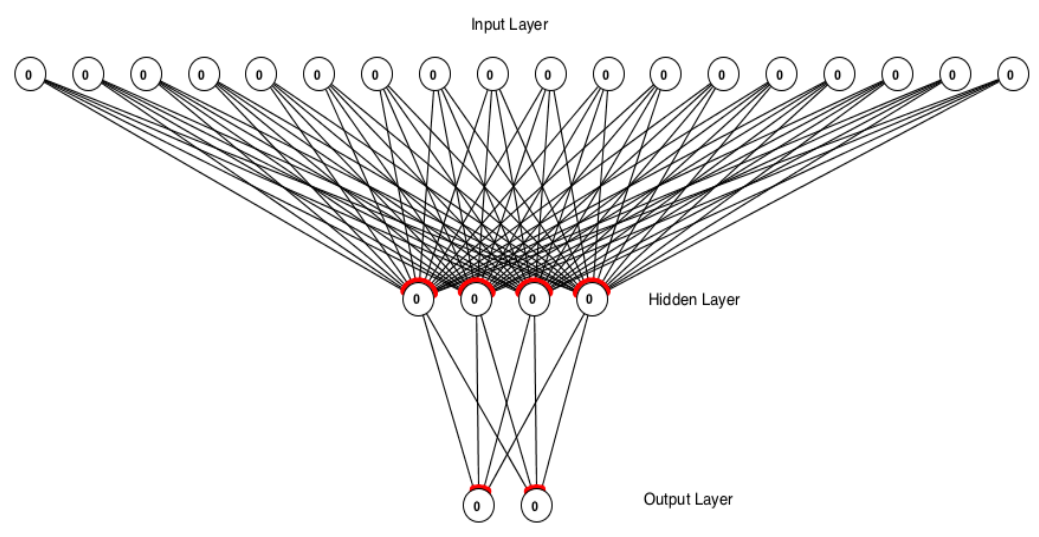

Figure 7. MLP Neural Network layers.

Figure 7 illustrates the topology of neurons in this network was distributed in an input layer with one neuron for each input parameter (attributes from 1 to 18), a hidden layer distributed in four neurons with randomized weights and two output neurons as result. This topology was defined after stable processing time on tests with generic dataset with higher amount of records.

\section{Training Neural Network}

The type of training method chosen to this first classification task is Supervised training, to intend minimize the error of classification through an iterative process. Supervised training is accomplished by providing to neural network a sample dataset with the anticipated outputs expected for each record of these samples. As the process of supervised training proceeds, the neural network is taken through a number of iterations, until the output of the neural network matches the anticipated output, with a reasonably small and predefined rate of error. Error rate we find to be appropriate to make the network well trained is set just before the training starts. In this classification task, the highest error rate was defined around 0.010 . The target limit defined 0.010 reached nearby 91 iterations and after reduction, keeps in stable converging with Mean Square Error approximately at: 0.0073344 .

\section{Testing Neural Networks}

In MLP implemented, all selected test records where identified correctly validating the training process. 


\begin{tabular}{|c|c|c|}
\hline \multicolumn{3}{|c|}{ Test Results } \\
\hline MLP & Total Records Tested & $\%$ Success \\
\hline $\begin{array}{c}\text { Classification of } \\
\text { Environment EE }\end{array}$ & 15 & $100 \%$ \\
\hline $\begin{array}{l}\text { Classification of } \\
\text { Environment GU }\end{array}$ & 15 & $100 \%$ \\
\hline
\end{tabular}

Table 2. Result for MLP tests

Table 2 contains the amount of and the result of each class tested. To environment classification test, 30 records selected from test set was successfully categorized by MLP, being 15 from EE class and 15 from GU class. With this process, the minimal Kernel engine becomes able to identify and classify locales.

\section{Mobile Application: Simulator of Multiagent model}

The mobile application built, contains the algorithms orchestrating communication with Kernel engine. Given its reduced functionalities, there is no interoperability from mobile application with student's university platform. The dynamic proposed to participants of experiment, was to indicate on application what are the next tasks to execute and, point desired resources to be used to accomplished it.

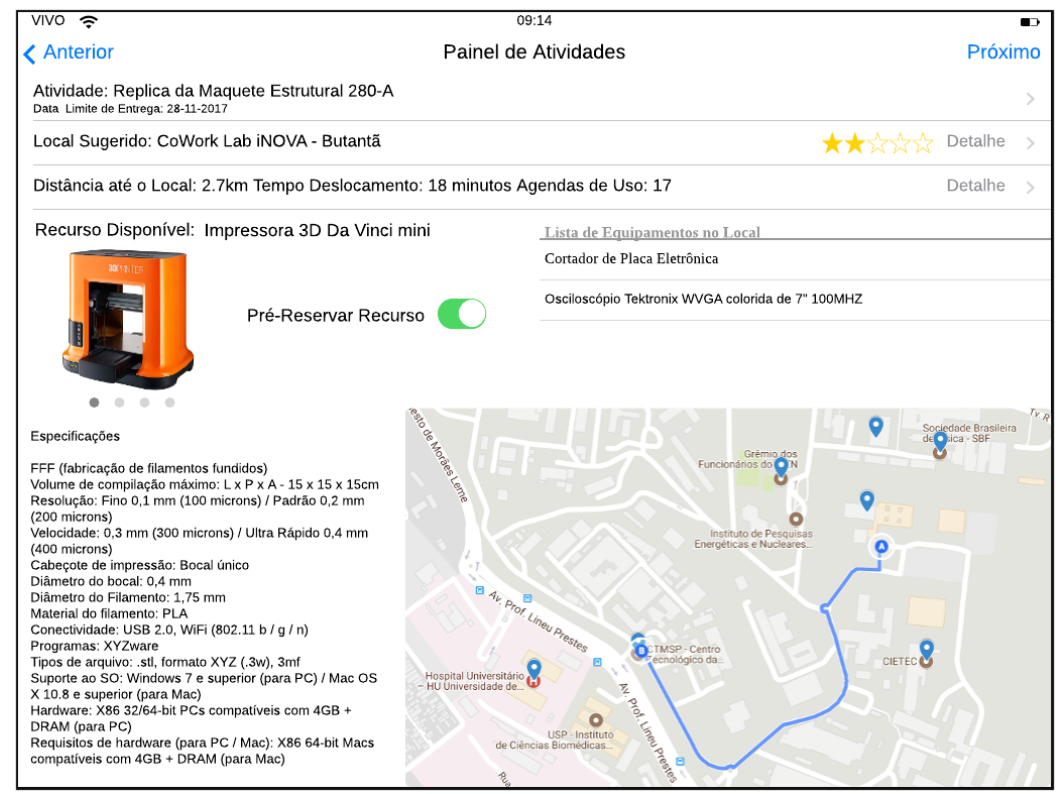

Figure 8. Mobile Application: routing recommendation interface

Figure 8 illustrates the main activity panel of mobile application. Simulating the local Agent behavior, this implementation provides the navigation presented through integration with map services, computing locally the distance and time to reach next locale. The definition of point $B$ presented on map as recommended destination, is defined by Kernel and queried by local Agent. This recommendation is based on a list of activities previously load into platform by 
students. The algorithms implemented in Kernel, considers the description of necessary resources point by students and their current location coordinates. If a student confirms the start of an activity and confirm the recommendation, the accept signal of suggestion is collected to rank Kernel process as assertive. The data of coordinates collected automatically in perimeter of suggested locale, confirms this process.

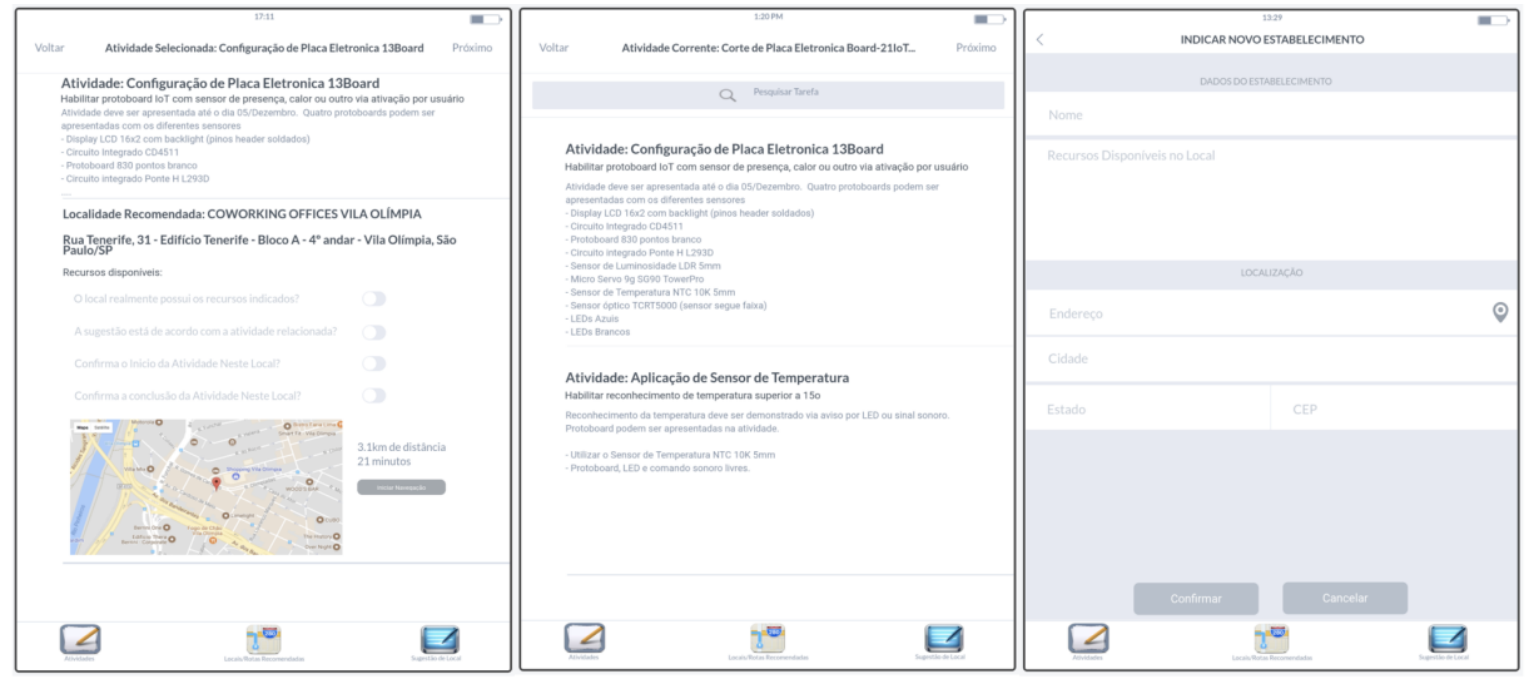

Figure 9. Capture of main interfaces

Figure 9 contains, in the sequence, the screens with activity start selection, the list of student's activities and the form to indicate a new locale. In the application interface, an evaluation feedback about the locale and its resources is available to students and makes part of rank computation. If students decide to execute the activity in an unknown location, the application provides a form to describe the new place, added by a check-in process where coordinates of locale are collected. Other data related to application use by students are collected to evaluate additional questions regarding time of application use, frequency of use and operation events like network issues, for example.

\section{RESULTS, CONCLUSIONS AND FURTHER WORK}

A detailed analysis on data collected by participants, demonstrates that given the 35 initial volunteers, 27 students followed the experiment until its expected ending. Tracking application use and navigation behavior, we identify that 16 students followed the route recommended by Kernel without location changes. Other 7 students changed the location suggested after issues with target resources and problems related to infrastructure. A special case of 4 students performed its activities indicating new locations not previously loaded into dataset. Without previous knowledge about the overall scope foreseen to this platform, students sent feedbacks at the end of each participation. They mentioned the absence of integration between the application provided and their educational platform, as an important functionality to be complemented. In these observations collected, students mentioned the effective resources reservation, and information about related costs if applicable on recommended 
locales, as desirable functionality to be added to application. In this group, 16 students suggested an integration between this application with Google Allo and Apple Siri services. All 27 students pointed this application as a desirable tool in online education platforms if provided communication with their teachers.

The feedback collected, reinforces the needs to elaborate the interactivity between the Kernel and context-aware environments, providing more information elements to student decisions.

This research, faces challenge related to security, identity and privacy information management that needs to be covered. Social Networks and physical places sharing educational content and data related to groups and individuals, requires a deep and clear view on ethic questions and right methods to manipulate this information.

The centric propose on this research, using artificial intelligence models to support the platform, requires an accurate analysis on which model of load, calibration and criteria of verification will be adequate to determine the refinement process of computational models proposed.

Technical questions regarding to architecture of platform implementation constitute an important phase to define the ideal setup to more flexible and evolve the proposed architecture. For this case, some aspects of reconfigurable and dynamic architectures will be investigated to improve the model. To support preserve the extensibility and evolving model as an open platform, a minimum set of patterns to computational maintenance, manageability and related aspects will be investigated and adequate to this research on its implementation process. Addressing points regarding information security and architectural patterns, the interoperability with educational platforms and context-aware environment can be stablished.

Context-Aware Computing definition and modeling must conform the target platform requirements considering the evolutionary environment and, to support reach this objective, a complementary study of ontology and common environment description will be covered during this research.

This initial experiment indicates the implementation viability of computational model proposed in the research. The experimental behavior using the strategy of separated implementation to process environment classification an activities routing and recommendation, allows the use of different computational models (external components or specialized platforms acting as modular services) in interaction with Kernel processing as proposed in this research conception.

The experiment is not conclusive in relation of definitive neural network topology and methods and, the better computational intelligence techniques to be applied on evolving model as its comprehensive form proposed on this research. The continuity of investigations will cover these theoretical details and 
technical aspects. The complexity of environment attribute identification and verification presents one of most relevant problems to solve and, consequently, determine the method to data normalization.

Conclusions initially obtained of current investigations and initial experiment provides base to evolve the research, considering the direction of educational platforms moving to cloud environment to attend growing of online courses and, consequently, the personification as a need in educational process. The interactive educator's perspective, contemplated in next phase of investigation, will analyze the approach to intermediate communication between actors involved in this dynamic. In parallel, points covered at current stage of investigation, will be improved according to new findings.

\section{REFERENCES}

Abowd, G., Dey, A., Brown, P., Davies, N., Smith, M., \& Steggles, P. (1999). Towards a better understanding of context and context-awareness. In Handheld and ubiquitous computing (pp. 304-307). Springer Berlin/Heidelberg.

Alshwaier, A., Youssef, A., \& Emam, A. (2012). A new trend for e-learning in KSA using educational clouds. Advanced Computing, 3(1), 81. Doi: https://doi.org/10.5121/acij.2012.3107.

Ally, M. (Ed.). (2009). Mobile learning: Transforming the delivery of education and training. Athabasca University Press.

Aouatef, C., Iman, B., \& Allaoua, C. (2014, August). Adaptive composition of services in context-aware ambient intelligent systems. In Proceedings of the 2014 International $C^{*}$ Conference on Computer Science \& Software Engineering (p. 26). ACM. Doi: https://doi.org/10.1145/2641483.2641535

Baldauf, M., Dustdar, S., \& Rosenberg, F. (2007). A survey on context-aware systems. International Journal of Ad Hoc and Ubiquitous Computing, 2(4), 263-277.

Basheer, I. A., \& Hajmeer, M. (2000). Artificial neural networks: fundamentals, computing, design, and application. Journal of microbiological methods, 43(1), 3-31. Doi: https:// doi.org/10.1016/s0167-7012(00)00201-3

Bellavista, P., Corradi, A., Fanelli, M., \& Foschini, L. (2012). A survey of context data distribution for mobile ubiquitous systems. ACM Computing Surveys (CSUR), 44(4), 24. Doi: https:// doi.org/10.1145/2333112.2333119

Chow, W. S., \& Chan, L. S. (2008). Social network, social trust and shared goals in organizational knowledge sharing. Information $\mathcal{E}$ management, 45(7), 458-465. Doi: https:// doi.org/10.1016/j.im.2008.06.007

Henricksen, K., \& Indulska, J. (2006). Developing context-aware pervasive computing applications: Models and approach. Pervasive and mobile computing, 2(1), 37-64. Doi: https://doi.org/10.1016/j.pmcj.2005.07.003

Hsu, M. H. (2008). A personalized English learning recommender system for ESL students. Expert Systems with Applications, 34(1), 683-688. Doi: https://doi.org/10.1016/j.eswa.2006.10.004

Ingersoll, G. M., Scamman, J. P., \& Eckerling, W. D. (1989). Geographic mobility and student achievement in an urban setting. Educational evaluation and policy analysis, 11(2), 143-149. Doi: https://doi.org/10.2307/1163781 
Kwon, O. B., \& Sadeh, N. (2004). Applying case-based reasoning and multi-agent intelligent system to context-aware comparative shopping. Decision Support Systems, 37(2), 199-213. Doi: https://doi.org/10.1016/s0167-9236(03)00007-1

Kwon, O., Choi, S., \& Park, G. (2005). NAMA: A context-aware multi-agent based web service approach to proactive need identification for personalized reminder systems. Expert Systems with Applications, 29(1), 17-32. Doi: https://doi.org/10.1016/j.eswa.2005.01.001

López-Nores, M., Blanco-Fernández, Y., Pazos-Arias, J. J., \& Gil-Solla, A. (2012). Property-based collaborative filtering for health-aware recommender systems. Expert Systems with Applications, 39(8), 7451-7457. Doi: https:// doi.org/10.1016/j.eswa.2012.01.112

Makris, P., Skoutas, D. N., \& Skianis, C. (2013). A survey on context-aware mobile and wireless networking: On networking and computing environments' integration. IEEE communications surveys \& tutorials, 15(1), 362-386. Doi: https://doi.org/10.1109/surv.2012.040912.00180

Maren, A. J., Harston, C. T., \& Pap, R. M. (2014). Handbook of neural computing applications. Academic Press.

Mitchell, M. (1998). An introduction to genetic algorithms. MIT press.

Ogata, H., \& Yano, Y. (2004). Context-aware support for computer-supported ubiquitous learning. In 2nd IEEE International Workshop on Wireless and Mobile Technologies in Education. Proceedings. (pp. 27-34). IEEE. Doi: https://doi.org/10.1109/wmte.2004.1281330

Rosaci, D., \& Sarné, G. M. (2010). Efficient Personalization Of E-Learning Activities Using A Multi-Device Decentralized Recommender System. Computational Intelligence, 26(2), 121-141. Doi: https://doi.org/10.1111/j.1467-8640.2009.00343.x

Santos, F. R. D. (2010). Comunidades Virtuais Baseadas Em Vídeo Digital: Uma Proposta de Conteúdo Adaptativo Pautada em Redes de Aprendizagem e Agentes Inteligentes (master's thesis). Mackenzie Presbyterian University, São Paulo, SP, Brazil. Retrieved from http://tede.mackenzie.br/jspui/handle/tede/1514

Samarasinghe, S. (2016). Neural networks for applied sciences and engineering: from fundamentals to complex pattern recognition. CRC Press.

Schilit, B., Adams, N., \& Want, R. (1994, December). Context-aware computing applications. In First Workshop on Mobile Computing Systems and Applications. (pp. 85-90). IEEE. Doi: https://doi.org/10.1109/mcsa.1994.512740

Silva, L., Mustaro, P. N., Stringhini, D., \& Silveira, I. F. (2006). Using Conceptual Lattices to Represent Fine Granular Learning Objects through SCORM MetaObjects. Electronic Journal of e-Learning, 4(2), 141-148.

Soldatos, J., Pandis, I., Stamatis, K., Polymenakos, L., \& Crowley, J. L. (2007). Agent based middleware infrastructure for autonomous context-aware ubiquitous computing services. Computer Communications, 30(3), 577-591. Doi: https://doi.org/10.1016/j.comcom.2005.11.018

Viel, C. C., Melo, E. L., Pimentel, M. D. G., \& Teixeira, C. A. (2013, November). Multimedia multi-device educational presentations preserved as interactive multi-video objects. In Proceedings of the 19th Brazilian symposium on Multimedia and the web (pp. 51-58). ACM. Doi: https:// doi.org/10.1145/2526188.2526211

Yang, S. J. (2006). Context aware ubiquitous learning environments for peer-to-peer collaborative learning. Educational Technology \& Society, 9(1), 188-201.

Zimmermann, A., Lorenz, A., \& Oppermann, R. (2007). An operational definition of context. Context, 7, 558-571. Doi: https://doi.org/10.1007/978-3-540-74255-5_42 